\title{
Design and Manufacture an Elastic Neutron Scattering Spectrometer at the Dalat Nuclear Reactor
}

\author{
Dang Hong Ngoc Quy, Pham Ngoc Son, Phan Bao Quoc Hieu, Trinh Van Cuong \\ Nuclear Research Institute, Dalat, Lam Dong, Vietnam \\ Email: danghongngocquy@gmail.com
}

How to cite this paper: Quy, D.H.N., Son, P.N., Hieu, P.B.Q. and Van Cuong, T. (2021) Design and Manufacture an Elastic Neutron Scattering Spectrometer at the Dalat Nuclear Reactor. Journal of Analytical Sciences, Methods and Instrumentation, 11, 23-28. https://doi.org/10.4236/jasmi.2021.113003

Received: August 24, 2021

Accepted: September 27, 2021

Published: September 30, 2021

Copyright $\odot 2021$ by author(s) and Scientific Research Publishing Inc. This work is licensed under the Creative Commons Attribution International License (CC BY 4.0). http://creativecommons.org/licenses/by/4.0/

\begin{abstract}
The objective of this study is to design an elastic neutron scattering system according to the angle with a sample using thermal neutron beam at the Dalat Nuclear Reactor (DNR). The system is used for research and training in the field of material structure analysis by neutron scattering and diffraction techniques. It is designed on the basis of inheriting the neutron measurement spectrometer systems at the DNR and the scattered neutron measurement systems in the world. The measuring system, which was installed at the horizontal channel 4 of the DNR, consists of 5-helium-3 detectors and a fully electronic system to record the scatter counts and a mechanical system with the possibility of rotating at $15^{\circ}-75^{\circ}$ angles. The constructed system is tested for evaluation of the accuracy, stability and reliability of the mechanical and electronic systems of moving detectors by angles.
\end{abstract}

\section{Keywords}

Neutron Scattering, Small-Angle Neutron Scattering (SANS), Elastic Neutron Cross Section

\section{Introduction}

The neutron scattering technique is playing a crucial part in determining properties and structures of solid, liquid, gaseous materials, nanomaterials, crystals, crystal lattice dynamics, etc. There are four neutron measurement techniques: transmission, inelastic scattering, elastic scattering, and echo-spin neutron. The elastic scattering technique is a measure of the intensity of scattered neutrons through angles to determine the properties and structure of materials. Diffraction, reflection, and Small-Angle Scattering Systems (SANS) operating effectively at JAEA 
of Japan, at Dubna JINR in Russia, etc., are also based on those methods [1] [2]. Neutron scattering systems are mainly designed and installed at research reactors such as JRR in Japan, IBR-2 in Dubna, IBR-2 in Dubna, Russia, MITR in the USA ... or other accelerators. There are four main types of neutron scattering: sample transmission, elastic neutron scattering, quasielastic or inelastic neutron scattering and spin-echo instrument [3]. In Vietnam, there are not any neutron scattering systems applied. In previous research, a small-scale scattering system was developed for evaluating the applicability of this technique at DNR. However, the results were not accurate as the system was measured and set up manually. For those reasons mentioned above, a scattered neutron measurement system has been designed and installed.

\section{Methodology}

\subsection{The Overall Design of Neutron Scattering}

The design model of the neutron scattering measurement system is shown in Figure 1 . The system is designed using 5 heli-3 detectors with pressure of 4 atm to increase the efficiency. Detectors are placed on a rotating mechanism around the sample target position and are automatically controlled to change the position of the detectors to record the neutron spectrum distributed at different angles after scattering with the sample target. A stepper motor is used and controlled automatically to increase the accuracy and angular stability. The neutron scattering measurement system consists of the following parts: Angle rotation mechanism system; Neutron measuring system; Radiation shielding system; Electronic control system.

\subsubsection{Angle Rotation Mechanism System}

The system is designed in the shape of a semicircle with a diameter of $2.0 \mathrm{~m}$. The

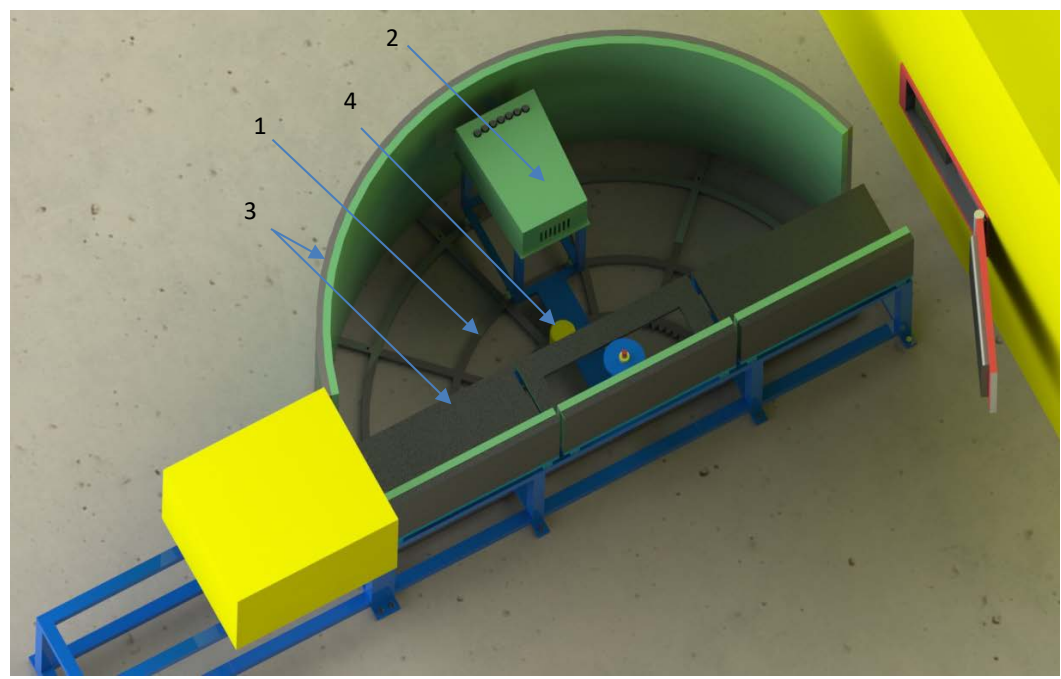

Figure 1. The design of the neutron scattering measurement system on the horizontal channel of the Dalat nuclear reactor. (1) Angle rotation mechanism system; (2) Neutron measuring system; (3) Radiation shielding system; (4) Electronic control system. 
circular arc is fabricated from U-shaped steel $200 \mathrm{~mm}$ wide, $75 \mathrm{~mm}$ high and 9.0 $\mathrm{mm}$ thick. The mechanical part moving the detector includes a mechanical system for the detector holder; a drive system rotates the detector holder at angle, a stepper motor and controller, Microcontroller (Pic 16f877) that controls the mechanical system via wireless RS232 communication, cooling fan, a small stepper motor to rotate the sample tray. The rotary system uses a small motor $(12 \mathrm{~V} / 17$ RPM) to rotate the sample tray and a large industrial motor (86BYGH450C) to rotate the entire probe system at angles.

\subsubsection{Neutron Measuring System}

The block diagram of electronic system is shown in Figure 2. The neutron scattering system consists of five He-3 LND Model 2528 counters [3] connected to a CANBERRA Model 2006 preamplifier [4] and powered by a $3 \mathrm{kV}$ CANBERRA Model 3002D high voltage block [5]. The signal from the preamplifier output is amplified and pulsed by the CANBERRA Model 2022 amplifier block [6]. The analog signal is then converted to a digital signal via the Multi-port II block [7] connected to a PC via the GENIE 2000 program [8]. The chamber containing the neutron detectors (He-3 counter) is made of high density polyethylene (HDPE) containing 5\% boron (Borated Polyethylene HD SWX-201 5\%) with the ability to absorb neutrons to minimize radiation dose. The detector chamber is located at a distance from the sample target so that the distance from the center of the detectors to the center of the sample target is $1 \mathrm{~m}$ to ensure the collimation of the scattered neutron beams from the sample target to the detector. In order to increase the recording performance as well as improve the angular resolution of the measuring system, the 5 neutron detectors are designed to be spaced at an angle of 2 degrees from the center of the target.

\subsubsection{Radiation Shielding System}

The neutron scattering system is installed in space with a rotation radius of $1 \mathrm{~m}$. Therefore, shielding of neutron and gamma radiation is essential. The radiation shielding system consists of two parts: a shield around the sample location and a shield behind the detector chamber.
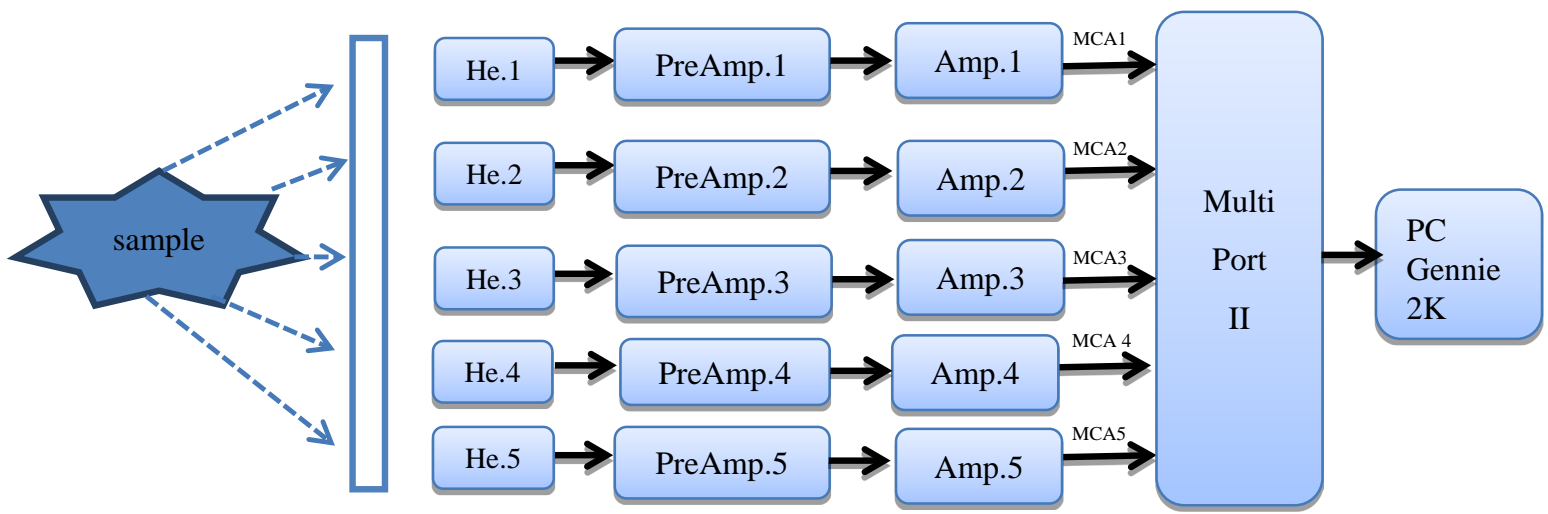

Figure 2. The block diagram of functional electronic blocks of neutron scattering measurement system. 
- The shielding around the sample location is composed of High Density Polyethylene (HDPE) sheets containing 5\% boron (SWX-201) $75 \times 34 \mathrm{~cm}$ in size, $3.0 \mathrm{~cm}$ thick to shield neutron and lead radiation, size $5 \times 40 \mathrm{~cm}$, thickness 3.0 $\mathrm{cm}$ outside for gamma radiation shielding.

- The shield behind the detector chamber consists of 2 HDPE sheets containing 5\% boron (SWX-201) high density polyethylene (SWX-201) with dimensions of $12.2 \times 12.2 \times 2.5 \mathrm{~cm}$, each. These shields are mounted on a steel frame with wheels for mobility.

\subsubsection{Electronic Control System}

The control system for the neutron scattering measurement system consists of a PIC controller board (PIC 16F886) [9] which controls two stepper motors for the angular detector movement mechanism and the sample rotation mechanism. The block diagram of the electronic system is shown in Figure 3.

\section{Results and Discussion}

The neutron scattering measurement system was installed and tested on the channel 4 Dalat nuclear reactor. To improve the accuracy when setting measuring angles for the scattering system, the project team designed and manufactured an automatic detector moving system and accompanying YAT control software. The YAT program is used to control the drive system to rotate in an angle. The interface of the program is shown in Figure 4.

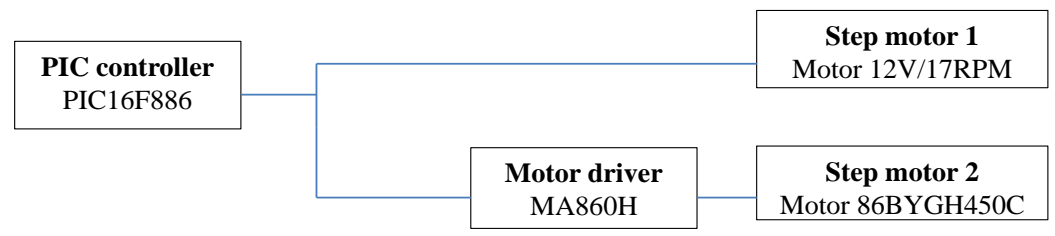

Figure 3. Control system block diagram.

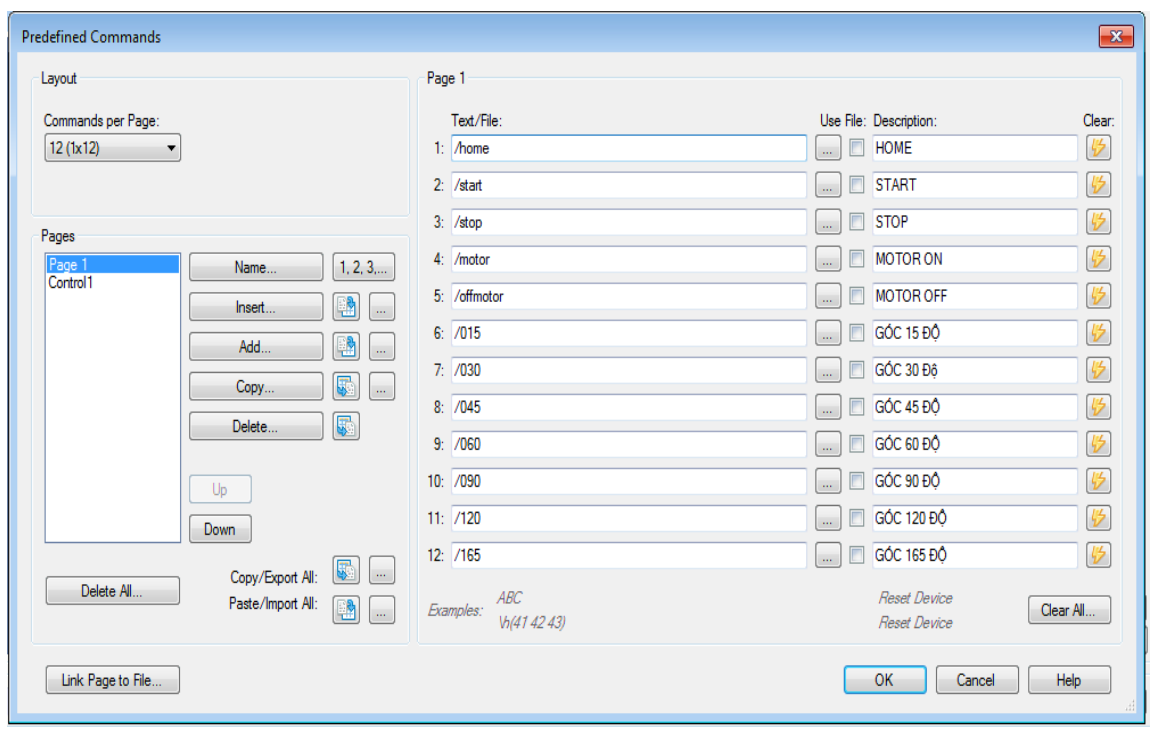

Figure 4. YAT control software to set preset angle value. 


\subsection{Check the Operation and Stability of the System}

To check the accuracy and stability of the motor and the rotary system, the test steps are performed in turn.

From the results in Table 1 , the rotation angle deviation is $<0.5 \%$, proving that the rotating mechanical system operates accurately, stably and reliably.

\subsection{Check the Operation of the Neutron Measurement Electronic System}

The Helium-3 counters are connected to a CANBERRA Model 2006 preamp and connected voltage $1000 \mathrm{~V}$ from a $3 \mathrm{kV}$ CANBERRA Model 3002D high voltage block. The preamplifier output signal is amplified and pulsed through a CANBERRA Model 2022 amplifier and connected to a Multi-port II block (6 units). The GENNIE2K program was used to acquire the spectrum.

In this experimental configuration, the reaction between thermal neutron and He-3 counter is shown as follows:

$$
{ }^{3} \mathrm{He}+\mathrm{n} \rightarrow{ }^{1} \mathrm{H}+{ }^{3} \mathrm{H}+764 \mathrm{keV}
$$

For proportional counter, the dead time of the measuring system is usually high $>10 \%$ due to the signal from gamma rays and pulse superposition of low energy region. To reduce the dead time of the measurement system, the LLD cut off of the amplifier is used. In this experiment, the LLD threshold set at $3 \%$ corresponds to a dead time of about $0.1 \%$.

The installation and testing of the entire system was carried out on the channel 4 of DNR. The result above shows the mechanical system rotates at angles;

Table 1. Test results of some parameters and stability of the mechanical system.

\begin{tabular}{ccccccc}
\hline Order & $\begin{array}{c}\text { Angle } \\
\text { (degree) }\end{array}$ & $\begin{array}{c}\text { Average rotational speed } \\
\text { (second/degree) }\end{array}$ & $\begin{array}{c}\text { Position } \\
\text { needle }\end{array}$ & $\begin{array}{c}\text { Graduated } \\
\text { ruler } \\
\text { (degree) }\end{array}$ & $\begin{array}{c}\text { Difference } \\
\text { (degree) }\end{array}$ & (degree) \\
\hline 1 & $0-180$ & 0.77 & 0.68 & 180.00 & 180.40 & 0.40 \\
2 & $0-15$ & 0.77 & 0.68 & 15.00 & 15.00 & 0.00 \\
3 & 30 & 0.77 & 0.68 & 30.00 & 30.00 & 0.00 \\
4 & 45 & 0.77 & 0.68 & 45.00 & 45.00 & 0.00 \\
5 & 60 & 0.77 & 0.68 & 60.00 & 60.00 & 0.00 \\
6 & 75 & 0.77 & 0.68 & 75.00 & 75.00 & 0.00 \\
7 & 90 & 0.77 & 0.68 & 90.00 & 90.00 & 0.00 \\
8 & 105 & 0.77 & 0.68 & 105.00 & 105.50 & 0.50 \\
9 & 120 & 0.77 & 0.68 & 120.00 & 120.50 & 0.50 \\
10 & 135 & 0.77 & 0.68 & 135.00 & 135.15 & 0.15 \\
11 & 150 & 0.77 & 0.68 & 150.00 & 135.20 & 0.20 \\
12 & 165 & 0.77 & 0.68 & 165.00 & 165.25 & 0.25 \\
13 & 180 & 0.77 & 0.68 & 180.00 & 180.40 & 0.40 \\
\hline
\end{tabular}


the electronic control system and the neutron measurement system with $5 \mathrm{He}-3$ counters operate accurately, stably and reliably.

Recommendations for future research directions will include as follows:

- Continue design shielding and reducing background;

- Measure some neutron beams with different energies.

- Currently, the scattering neutron in a cylinder with a diameter equal to the detector diameter of $2.5 \mathrm{~cm}$, which has not been collimated. The scattered neutron can only reach the detector in a narrow gap. Therefore; angular measurement error also need to be corrected.

\section{Conclusion}

A neutron scattering spectrometer system is fully installed at channel 4 of the Dalat nuclear reactor. However, there is still no scattering data when measuring with the samples. The research groups only checked the stability of the mechanical and electronic systems. The aim in the near future is to measure the elastic scattering cross-section with different samples and calculate the elastic neutron scattering cross-section.

\section{Conflicts of Interest}

The authors declare no conflicts of interest regarding the publication of this paper.

\section{References}

[1] Anh, T.T., Son, P.N., Tan, V.H., Khang, P.D. and Hoa, P.C. (2014) Characteristics of Filtered Neutron Beam Energy Spectra at Dalat Reactor. World Journal of Nuclear Science and Technology, 4, 96-102. https://doi.org/10.4236/wjnst.2014.42015

[2] Iijima, T., Mukaiyama, T. and Shirakata, K. (1971) Measurement of Fast Neutron Spectrum with Helium-3 Proportional Counter. Journal of Nuclear Science and Technology, 8, 192-200. https://doi.org/10.1080/18811248.1971.9735315

[3] Ibaraki, M., Baba, M., Miura, T., Hirasawa, Y., Nauchi, Y., Nakashima, H., Meigo, S.-I., Iwamoto, O. and Tanaka, S. (2000) Experimental Method for Neutron Elastic Scattering Cross-Section Measurement in 40-90 MeV Region at TIARA. Nuclear Instruments and Methods in Physics Research A, 446, 536-544. https://doi.org/10.1016/S0168-9002(99)01274-7

[4] Stokes, R. (2005) Helium 3 Detector 207 Formation. General Electric Company.

[5] Canberra (2007) Model 2006 Proportional Counter Preamplifier Datasheet.

[6] Canberra (2010) 3002D High Voltage Power Supply Datasheet.

[7] Canberra (2010) 2022 Amplifier Datasheet.

[8] Canberra (2017) Canbera Multiport II Datasheet.

[9] Microchip Technology, Inc. (2007) Pic16F886 Datasheet. 